\title{
Trigeminal sensory symptoms associated with hydrocephalus
}

\author{
R. S. M A U R I C E - W I L L I A M S A N D J O H N P I L L I N G \\ From the Department of Neurosurgery and Neurology, St. Bartholomew's Hospital, London
}

SUMMARY In three patients, trigeminal sensory symptoms remitted with treatment of associated hydrocephalus, two with facial pain resembling trigeminal neuralgia, and one with facial paraesthesiae. The pathological basis of these phenomena and their relationship to idiopathic trigeminal neuralgia are discussed.

Facial pain, similar in character to trigeminal neuralgia, can follow involvement of the trigeminal nerve sensory root either by an adjacent mass lesion or by a plaque of demyelination, but the occurrence of this type of pain as a symptom of hydrocephalus does not appear to have been described previously. We report here two instances of hydrocephalus associated with paroxysmal facial pain where relief of the hydrocephalus by a CSF shunt led to remission of the pain. In a third patient with hydrocephalus, the severity of facial numbness and paraesthesiae of uncertain aetiology fluctuated with the functioning of a shunt.

\section{Case 1}

\section{(MH G 28317)}

In 1964 a 47 year old nurse presented with a three year history of deteriorating memory, neck pain, and pain and weakness in the left arm. At the ages of 12 and 44 years she had had episodes of headache, vomiting, and double vision lasting for three months, and at the age of 31 years had developed a left hemiparesis which persisted for several months. The onset of this hemiparesis had been abrupt with a period of unconsciousness lasting many hours. For at least 20 years she had suffered from occasional fronto-occipital headaches.

On examination there was no gross abnormality of her mental state. She had pale optic discs with slightly blurred margins, thought to be indicative of secondary optic atrophy, and had minimal weakness of the left side of the face and the left leg.

Accepted 1 March 1977
A skull radiograph showed erosion of the dorsum sellae. Lumbar pneumoencephalography showed gross ventricular dilatation but air failed to rise over the surfaces of the cerebral hemispheres. The lumbar CSF pressure was $175 \mathrm{~mm}$, and the CSF contained 3 lymphocytes per $\mathrm{mm}^{3}$, a protein level of $0.3 \mathrm{~g} / 1$ and a negative WR. The Lange curve was normal. She was diagnosed as having a communicating hydrocephalus, and a right sided Holter ventriculo-atrial shunt was inserted.

Postoperatively her symptoms improved for one month when she deteriorated again. In addition she now also complained of an ache behind the right eye with stabs of pain radiating over the right side of the forehead. The valve system was revised and a blocked ventricular catheter replaced. After this operation the ache behind the eye persisted but the sharp stabs of pain ceased. Her other symptoms again resolved.

She then remained well for seven years until 1971 when headaches and intellectual deterioration appeared again. At the same time pains on the right side of the face also recurred. They were described as being sudden excruciating pains beginning in the right cheek and radiating through the right orbit to the right ear and the vertex. The pains would last under 30 seconds and leave in their wake a dull right sided headache. There were no trigger spots or precipitating factors. On examination her gait was unsteady and she tended to veer to the left. She had a mild left hemiparesis and dulling of sensation on the left side of the face. Her valve chamber was non-compressible, and lumbar CSF pressure was $190 \mathrm{~mm}$ of water. After replacement of her valve system with a ventriculoperitoneal shunt her symptoms once more improved, and her facial pain disappeared. 
Case 2

(SBH 562934)

A 22 year old West Indian girl presented with a two year history of shooting pains in the left lower jaw. They were momentary, very severe pains brought on by eating, brushing her teeth, or by a cold wind. Carbamazepine $200 \mathrm{mg}$ four times daily controlled the pain initially, but as time went on it became less effective and eventually, despite it, she suffered from several bouts of pain each day.

At the age of 18 months she had had meningitis which had left her blind in the left eye and deaf in the right ear. Her subsequent intellectual development appeared normal and she worked as a clerk for a music publisher.

On examination her mental state was normal. Head circumference was $610 \mathrm{~mm}$. She had bilateral pallor of the optic discs and uncorrected visual acuity was J.1 (right) and perception of movement (left). The right ear was completely deaf and showed no caloric responses, and she had a slight right facial nerve palsy. She had no other neurological signs apart from a slight unsteadiness of heel-toe walking.

Skull radiography showed no abnormality other than slight expansion of the vault. A right carotid angiogram showed marked enlargement of the lateral ventricle. On lumbar pneumoencephalography, no air entered the ventricular system but some passed above the tentorium to outline a widespread pericallosal sulcus. The lumbar cerebrospinal fluid had a protein content of $0.2 \mathrm{~g} / 1$ and 6 lymphocytes $/ \mathrm{mm}^{3}$. No abnormal cells were seen in the centrifuged deposit. Blood serology for syphilis was negative.

It was thought that she had a compensated hydrocephalus caused by post-meningitic obstruction of the outlets of the fourth ventricle, and that the facial pain could be a false localising symptom of this. A Holter ventriculo-atrial shunt with an opening pressure of $80 \mathrm{~mm}$ of water was inserted. No carbamazepine was given postoperatively but her neuralgia ceased from the time of the operation. Twelve months later her valve was pumping well, she was taking no medication, and had remained totally pain free.

\section{Case 3}

(SBH 566287)

A 49 year old previously fit woman was admitted with a 10 month history of a cold sensation over the right cheek. For two months she had had an unsteadiness of gait and some loss of mental concentration.
Examination of her mental state showed no abnormality, but she had dulling of sensation over the right trigeminal territory, maximal in the second division. Trigeminal motor function and the corneal reflexes were normal. The only other neurological abnormality was unsteady gait, although coordination of the limbs separately tested was normal.

A skull radiograph, EEG, and isotope brain scan were normal but a left carotid angiogram showed slight dilatation of the lateral ventricles. Vertebral angiography was thought to show displacement of vessels away from the right side of the posterior fossa, but lumbar pneumoencephalography showed no definite abnormality. The lumbar CSF contained no cells, had negative serology, and a protein content of $0.23 \mathrm{~g} / 1$. Audiograms were normal, but the caloric responses were absent on the right.

A thorough exploration of the posterior fossa and right cerebellopontine angle revealed nothing abnormal. One month later she developed headaches and a tense fullness of the posterior fossa surgical decompression. The numbness over the right cheek had disappeared after the operation but now returned. A diagnosis was made of postoperative communicating hydrocephalus and a Holter ventriculo-atrial shunt was inserted.

After this, her headache, gait unsteadiness, and facial numbness were completely relieved for 10 weeks when all her symptoms and her original neurological signs returned. At the same time the decompression once more became tense and the shunt chamber was noted to be filling very slowly. Revision of the shunt led to partial improvement of the facial paraesthesiae and abnormal gait but the decompression remained full. A further shunt revision caused the decompression to become quite slack, and complete resolution of both the facial and gait symptoms took place.

\section{Discussion}

By convention, trigeminal neuralgia is regarded as an 'idiopathic' condition, but in a certain number of cases there appears to be irritation of the sensory root of the trigeminal nerve in the posterior fossa by an extrinsic lesion. Estimates of the proportion of cases in which this is so have ranged as high as $11 \%$ (Dandy, 1934), and where there is an associated ipsilateral hemifacial spasm ('tic convulsif') a causative lesion will probably be found in every case (Maurice-Williams, 1973). Even in those cases, the majority, where no gross cause can be found, the underlying pathological basis for the neuralgia probably lies in the sensory 
root and Gasserian ganglion, as electron microscope studies have shown consistent abnormalities at these sites (Beaver, 1967). The consensus of modern opinion is that the cause of trigeminal neuralgia type facial pain probably always lies distal to the trigeminal root entry zone of the brain stem (Kerr, 1970).

If this is so, it seems reasonable to postulate that in the cases reported here the basis for the paroxysmal facial pain and paraesthesiae lay in the sensory root, and was possibly due to stretching of the root by distal displacement of the brain stem due to the hydrocephalus. It has been shown that quite marked downward shift of the brain stem can take place in hydrocephalus, sufficient in some cases to cause an overfolding of the neuraxis at the cervicomedullary junction (Emery, 1967). When such shift occurs the stem end of the sensory root will be pulled downwards while the distal end is held fixed by the semilunar ganglion. Olivecrona (1947) suggested, indeed, that such traction might underlie many cases of trigeminal neuralgia. If this were so, however, one might expect to find trigeminal false localising signs occurring frequently when a significant shift of intracranial structures has taken place. On the contrary, it is most unusual for the fifth nerve to be so involved. So far as we can discover, trigeminal neuralgia has not been reported before as a symptom of hydrocephalus. In 124 cases of benign intracranial hypertension, no fifth nerve involvement was reported (Johnston and Paterson, 1974), and in a study of false localising signs in 250 intracranial meningiomas, in only eight instances was the trigeminal nerve so affected (Gassel, 1961).

From a study of the anatomy one might suppose that a nerve with such a relatively short course across the subarachnoid space as the fifth sensory root would be readily stretched by quite a minor degree of shift of the brain stem. On the other hand, as Collier (1904) pointed out, it is the cranial nerves with the most rostrocaudal axes, such as the sixth, which most commonly present false localising signs, while the more transversely running nerves, such as the seventh and eighth, are seldom affected. The fifth cranial nerve occupies an intermediate position in this respect. Collier also maintained that cranial nerve palsies as false localising signs appeared to be more common in the early days of neurology, but that as knowledge advanced the diagnosis of cerebral masses tended to be made before the occurrence of marked shift and traction of the intracranial structures.

Gardner (1970) has suggested that the cause of trigeminal neuralgia may be short-circuiting of electrical impulses between fibres of a similar size in the sensory root which are approximated by either loss of myelin or by external pressure. Such a theory accounts for the paroxysmal nature of the pain, the increased incidence in old age and demyelinating disease, and the production of the neuralgia by extrinsic masses. It would also account for the pain occurring from stretching of the nerve, as in hydrocephalus, when the fibres would be held more tightly together, especially at the point where the root angulates over the petrous ridge at the entrance to Meckel's cave. Angulation of the root will be greater if the petrous ridge is slightly higher on one side of the head than the other, and Gardner (1970) has found that where there is asymmetry of the petrous ridges in cases of trigeminal neuralgia the pain is present on the side of the higher ridge in three times as many cases. Gardner and Dohn (1966) reported bilateral trigeminal neuralgia occurring in a case of basilar impression, where again there might be stretching of the trigeminal sensory roots.

In the third case reported here, there was no facial pain but instead unilateral facial numbness accompanied by some unsteadiness of gait and slight intellectual change. The cause of these original symptoms remains obscure, but after a negative posterior fossa exploration the patient developed a communicating hydrocephalus and her symptoms, including the facial numbness, fluctuated with the varying efficacy of a shunting system. One could speculate as to whether the initial symptoms were in fact due to an incipient disturbance of CSF flow, as hydrocephalus would account for them, including the gait disturbance (Maurice-Williams, 1974), and even before operation there was slight ventricular dilatation.

We would like to thank $\mathrm{Mr} \mathrm{J}$. Andrew and Mr R. C. Connolly, for permission to report these cases which were treated under their care.

\section{References}

Beaver, D. L. (1967). Electron microscopy of the Gasserian ganglion in trigeminal neuralgia. Journal of Neurosurgery, 26, 138-150.

Collier, J. (1904). The false localising signs of intracranial tumour. Brain, 27, 490-508.

Dandy, W. E. (1934). Concerning causes of trigeminal neuralgia. American Journal of Surgery, 24, 447470.

Emery. J. L. (1967). Kinking of the medulla in children with acute cerebral oedema and hydrocephalus and its relationship to the dentate liga- 
ments. Journal of Neurology, Neurosurgery, and Psychiatry, 30, 267-275.

Gardner, W. J. (1970). Trigeminal neuralgia. In Trigeminal Neuralgia. Georg Thieme Verlag: Stuttgart.

Gardner, W. J., and Dohn, D. E. (1966). Trigeminal neuralgia, hemifacial spasm, Paget's disease. Brain, 89, 555-562.

Gassel, M. M. (1961). False localising signs. Archives of Neurology (Chicago), 4, 526-554.

Johnston, I., and Paterson, A. (1974). Benign intracranial hypertension-1: diagnosis and prognosis Brain, 97, 289-300.
Kerr, F. W. L. (1970). Peripheral versus central factors in trigeminal neuralgia. In Trigeminal Neuralgia. Georg Thieme Verlag: Stuttgart.

Maurice-Williams, R. S. (1973). Tic convulsif: the association of trigeminal neuralgia and hemifacial spasm. Postgraduate Medical Journal, 49, 742-745.

Maurice-Williams, R. S. (1974). Mechanism of production of gait unsteadiness by tumours in the posterior fossa. Journal of Neurology, Neurosurgery, and Psychiatry, 38, 143-148.

Olivecrona, H. (1947). The surgery of pain. Acta Psychiatrica Scandinavica, Supplement, 46, 268280. 